\title{
Childhood abuse and deprivation are associated with distinct sex-dependent differences in brain morphology
}

\author{
Daphne Everaerd*,1,2 , Floris Klumpers ${ }^{1,3}$, Marcel Zwiers', Tulio Guadalupe ${ }^{4}$, Barbara Franke ${ }^{1,2,5}$, \\ Iris van Oostrom ${ }^{1,2}$, Aart Schene ${ }^{1,2}$, Guillén Fernández ${ }^{1,3}$ and Indira Tendolkar 1,2,6 \\ 'Donders Institute for Brain, Cognition and Behaviour, Radboud University, Nijmegen, The Netherlands; '2Department of Psychiatry, Radboud \\ University Medical Center, Nijmegen, The Netherlands; ${ }^{3}$ Department for Cognitive Neuroscience, Radboud University Medical Center, Nijmegen, \\ The Netherlands; ${ }^{4}$ Language and Genetics Department, Max Planck Institute for Psycholinguistics, Nijmegen, The Netherlands; ${ }^{5}$ Department of \\ Human Genetics, Radboud University Medical Center, Nijmegen, The Netherlands; 'LVR Clinics of Psychiatry and Psychotherapy, University \\ Medical Center, University of Essen Duisburg, Essen, Germany
}

Childhood adversity (CA) has been associated with long-term structural brain alterations and an increased risk for psychiatric disorders. Evidence is emerging that subtypes of CA, varying in the dimensions of threat and deprivation, lead to distinct neural and behavioral outcomes. However, these specific associations have yet to be established without potential confounders such as psychopathology. Moreover, differences in neural development and psychopathology necessitate the exploration of sexual dimorphism. Young healthy adult subjects were selected based on history of CA from a large database to assess gray matter (GM) differences associated with specific subtypes of adversity. We compared voxel-based morphometry data of subjects reporting specific childhood exposure to abuse $(n=127)$ or deprivation $(n=126)$ and a similar sized group of controls $(n=129)$ without reported CA. Subjects were matched on age, gender, and educational level. Differences between CA subtypes were found in the fusiform gyrus and middle occipital gyrus, where subjects with a history of deprivation showed reduced GM compared with subjects with a history of abuse. An interaction between sex and CA subtype was found. Women showed less GM in the visual posterior precuneal region after both subtypes of CA than controls. Men had less GM in the postcentral gyrus after childhood deprivation compared with abuse. Our results suggest that even in a healthy population, CA subtypes are related to specific alterations in brain structure, which are modulated by sex. These findings may help understand neurodevelopmental consequences related to $C A$.

Neuropsychopharmacology (2016) 4I, I7I6-1723; doi: I0.1038/npp.20I5.344; published online 30 March 2016

\section{INTRODUCTION}

Childhood adversities (CAs) are among the most consistently documented risk factors for psychiatric disorders (Cuijpers et al, 2011; McLaughlin et al, 2012). They are in particular linked to affective, addiction, and personality disorders and possibly associated with distinct phenotypes within these disorders (Gilbert et al, 2009; Heim et al, 2010; McLaughlin et al, 2012). To learn more about the effects of these adversities on brain structure, it is essential to study healthy individuals where the consequences of $\mathrm{CA}$ are not confounded by the possible effects of psychopathology (Dannlowski et al, 2012). Indeed such studies have highlighted numerous long-term structural differences in the brain related to CA (Dannlowski et al, 2012; Lim et al, 2014; Lupien et al, 2009). Most consistently, changes in gray matter

*Correspondence: Dr D Everaerd, Donders Institute for Brain, Cognition and Behaviour, Radboud University Nijmegen, PO Box 9I0I, 6500 HB Nijmegen, The Netherlands, Tel: +3I 02436 68488, Fax: +3। 02436 68488, E-mail: Daphne.Everaerd@radboudumc.nl Received II June 2015; revised 21 September 2015; accepted 16 October 2015; accepted article preview online 18 November 2015
(GM) volume in the prefrontal cortex, sensory association cortices, anterior cingulate gyrus, the amygdala, hippocampus, insula, striatum, and the cerebellum have been found in healthy individuals when compared with controls with no history of adverse childhood experiences (Dannlowski et al, 2012; Edmiston et al, 2011).

Recently, it has been suggested that different types of adverse childhood experiences may lead to distinct morphological alterations in the brain, possibly corresponding to different types of psychopathology in adulthood (Humphreys and Zeanah, 2014; McLaughlin et al, 2014a; Sheridan and McLaughlin, 2014). For example, cortical thinning in the somatosensory cortex representing the female genital area was found exclusively in women that had experienced sexual abuse compared with other forms of childhood trauma (Heim et al, 2013). In subjects with a specific history of emotional abuse, thinning in areas involved in emotion processing such as the medial prefrontal cortex and precuneus have been found (Heim et al, 2013; van Harmelen et al, 2010). In addition, it was proposed that a distinction could also be made between adversity in the form of deprivation, such as absence of the expected social input, 
and direct threat, such as in active abuse. These two types of adversity potentially lead to different (neural) outcomes (Edmiston et al, 2011; Humphreys and Zeanah, 2014; McLaughlin et al, 2014a; Sheridan and McLaughlin, 2014). Deprivation could lead to changes in the association cortex, involved in higher cognitive and social processes, whereas abuse could give rise to alterations in circuits involved in emotional learning (McLaughlin et al, 2014a; Sheridan and McLaughlin, 2014). Although these approaches seem promising, practical problems such as co-existing psychopathology and the frequent co-occurrence of different subtypes of adversity have complicated the understanding of their specific impact on neural development.

Our first aim was therefore to assess healthy subjects for specific regional GM volume differences corresponding to different types of CA. To this end, we selected subjects from a database of more than 2700 subjects by their reported history of specific childhood events. We then created two matched groups that had experienced items from either the 'abuse' or 'deprivation' categories. Because of the healthy nature of our population, we also included relatively mild indicators of deprivation, such as death of a close relative (eg, caring grandparent).

We hypothesized that subjects with a history of deprivation would specifically show GM reductions compared with a matched control group in somatosensory brain regions and association cortex (McLaughlin et al, 2014a; Sheridan and McLaughlin, 2014). Subjects with a history of abuse were hypothesized to differ from the controls especially in brain regions involved in emotion processing and emotional learning, such as the amygdala and hippocampus, ventromedial and dorsolateral prefrontal cortex, and the precuneus (Heim et al, 2013; McLaughlin et al, 2014a; Sheridan and McLaughlin, 2014; van Harmelen et al, 2010).

An important secondary question was to find out whether the two distinct types of CA were associated with different GM correlates in the two sexes. Girls and boys have been found to show distinct regional developmental trajectories of GM during childhood and adolescence (Lenroot and Giedd, 2010; Rijpkema et al, 2011). The occurrence of adverse events during these periods could therefore have gender-dependent associations with GM in specific brain regions (Tottenham and Sheridan, 2009). Moreover, the type of event could also have a different impact. Importantly, men and women not only differ in prevalence of affective disorders, but also in their etiological pathways to depression (Kendler and Gardner, 2014). For example, men seem more vulnerable to (sexual) abuse, life events, and instrumental problems, whereas for women, problems relating to interpersonal warmth and relationships have greater impact (Kendler and Gardner, 2014). Finally, men have often been considerably underrepresented in the majority of studies looking into childhood events, making it difficult to generalize these results to both sexes, whereas the prevalence of CA in men is substantial (Edwards et al, 2003). We therefore hypothesized that sex could interact with the type of CA in its effect on GM structure.

\section{MATERIALS AND METHODS}

\section{Participants}

The study was part of the Cognomics Initiative's Brain Imaging Genetics (BIG) project at the Donders Institute for Brain, Cognition and Behavior of the Radboud University in Nijmegen, the Netherlands (www.cognomics.nl). Participants were screened before participation in this study by self-reported questionnaires. They were excluded if they had a history of somatic disease potentially affecting the brain, current or past psychiatric or neurological disorder, medication (except hormonal contraceptives) or illicit drug use during the past 6 months, history of substance abuse, current or past alcohol dependence, pregnancy, lactation, menopause or MRI contraindications. A total of 2737 subjects was included in the BIG project database at the time of our analysis.

Subjects were specifically selected for the current analysis based on their history of CAs. An age limit (age 18-35 years) was set to homogenize the groups. Of note, this is also the age range of onset for most forms of CA-related psychopathology (Lupien et al, 2009). Specific types of adversities were assessed using an adapted version of the 'List of Threatening Life Events' (Brugha et al, 1985). Participants were asked whether they had experienced a list of predefined events (a) before the age of 16 years and/ or (b) at or after the age of 16 years (Supplementary Table S1). Three groups were based on CA type: an 'abuse group', a 'deprivation group', and a control group. Subjects were assigned to the abuse group if (i) they reported any verbal, physical, and/ or sexual abuse before the age of 16 years and (ii) did not report any items indicating deprivation before 16 years. These deprivation items consisted of a history of separation from parent, severe financial problems, health problems of a close relative, and/ or death of a close relative. The latter two are not explicitly mentioned in common definitions of childhood deprivation (eg, (Sheridan and McLaughlin, 2014)), however are considered here as indicators that a child was deprived of expected social input (eg, death of a close relative that most likely resulted in the absence of a caregiver). Importantly, we only included subjects who indicated a history of separation from parent under 16 years, as separation from parent later in life does not necessarily indicate deprivation (eg, leaving home to study abroad). Subjects who reported any items indicating deprivation before the age of 16 years and did not report any lifetime abuse were assigned to the deprivation group. Subjects in the control group did not report any items before age 16 or any lifetime abuse.

The abuse group was the smallest group $(n=131)$ in our sample. Therefore, subjects from the two other groups were matched to the abuse group based on age, sex, and educational level to obtain equal group sizes. This gave us a subset of 393 subjects (131 per group) for our study. After the exclusion of 11 subjects due to insufficient data quality for VBM analysis or missing data, we had a final sample of 382 subjects (167 men and 215 women) for this analysis (Table 1). The mean (SD) age was 22.1 (3.7) years for men and 22.0 (3.4) years for women. 
Table I Characteristics of the Study Population

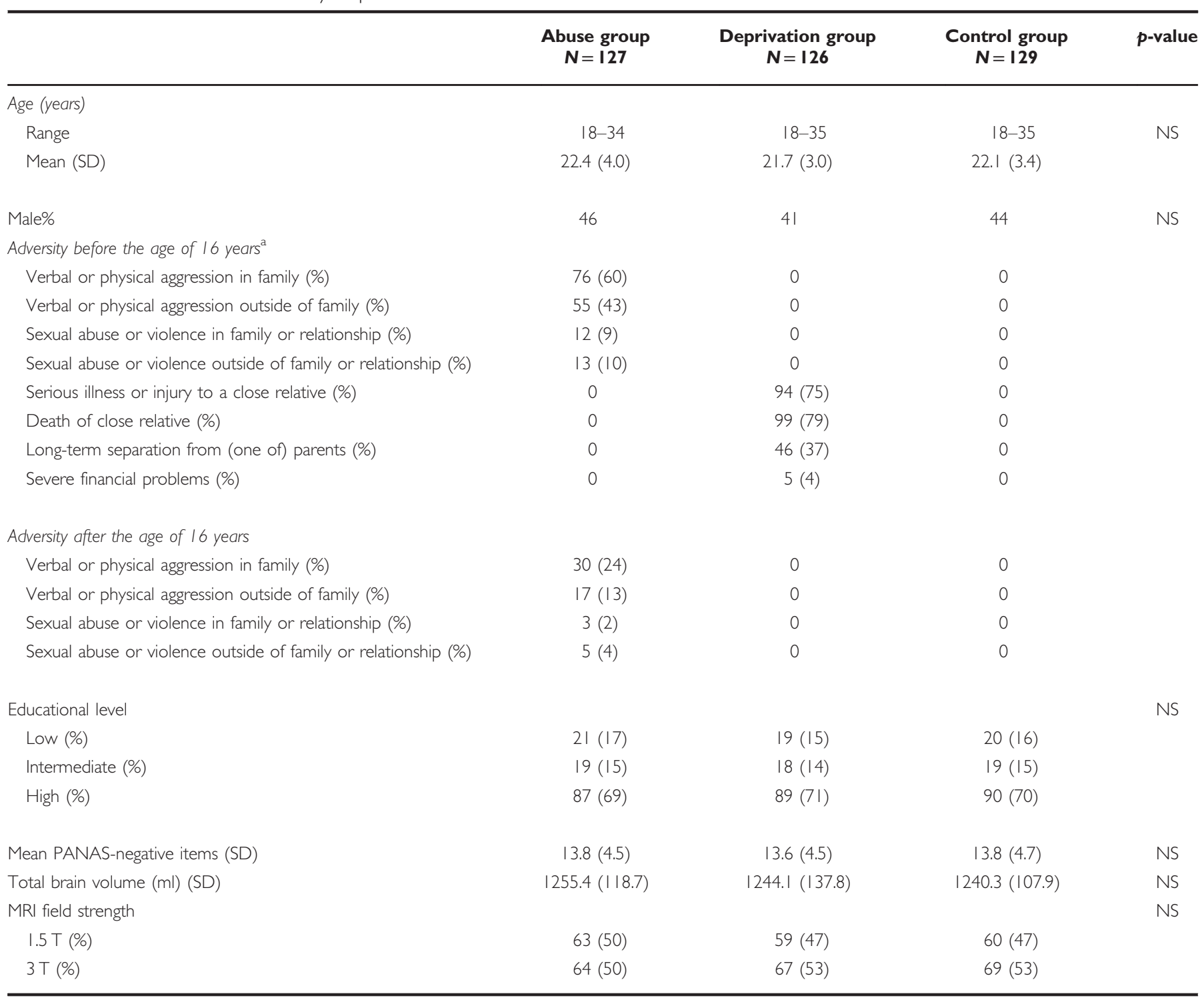

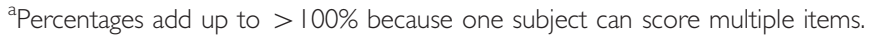

\section{Affect Rating}

The negative affect scale of the Positive Affect and Negative Affect Schedule (PANAS) was used to assess negative affect at the time of filling in the life-event questionnaire (Watson et al, 1988). Negative affect was entered as a covariate in our analysis to correct for possible recollection bias due to current affective state. When not correcting for PANAS scores, the effect of life events could be overestimated, as those individuals with currently lower mood may also be more likely to report negative life events (Chan et al, 2007). We also conducted our main analysis without PANAS correction, which enabled us to verify whether this covariate influenced our findings.

\section{MRI Acquisition}

Anatomical T1-weighted MRI data were acquired at the Donders Centre for Cognitive Neuroimaging. All scans covered the entire brain and had a voxel size of $1 \times 1 \times 1 \mathrm{~mm}^{3}$.

For $48 \%(n=182)$ of the subjects, images were acquired at $1.5 \mathrm{~T}$ Siemens Sonata or Avanto scanners (Siemens, Erlangen, Germany), using small variations to a standard T1-weighted 3D MPRAGE sequence (repetition time (TR) $2300 \mathrm{~ms}$, inversion time (TI) $1100 \mathrm{~ms}$, echo time (TE) $3.03 \mathrm{~ms}$, 192 sagittal slices, field of view $256 \mathrm{~mm}$ ). These variations included a TR/TI/TE/slices of 2730/1000/2.95/176, 2250/850/2.95/176, 2250/850/3.93/176, 2250/850/3.68/176, and the use of GRAPPA parallel imaging with an acceleration factor of 2 .

For all other subjects images were acquired at $3 \mathrm{~T}$ Siemens Trio, TimTrio, or Skyra scanners (Siemens), using small variations to a standard T1-weighted 3D MPRAGE sequence (TR $2300 \mathrm{~ms}$, TI $1100 \mathrm{~ms}$, TE 3.93 ms, 192 sagittal slices, field of view $256 \mathrm{~mm}$ ). These variations included TR/TI/TE/slices of 2300/1100/3.03/192, 2300/1100/2.92/192, 2300/1100/ $2.96 / 192, \quad 2300 / 1100 / 2.99 / 192,1940 / 1100 / 3.93 / 176$, and 


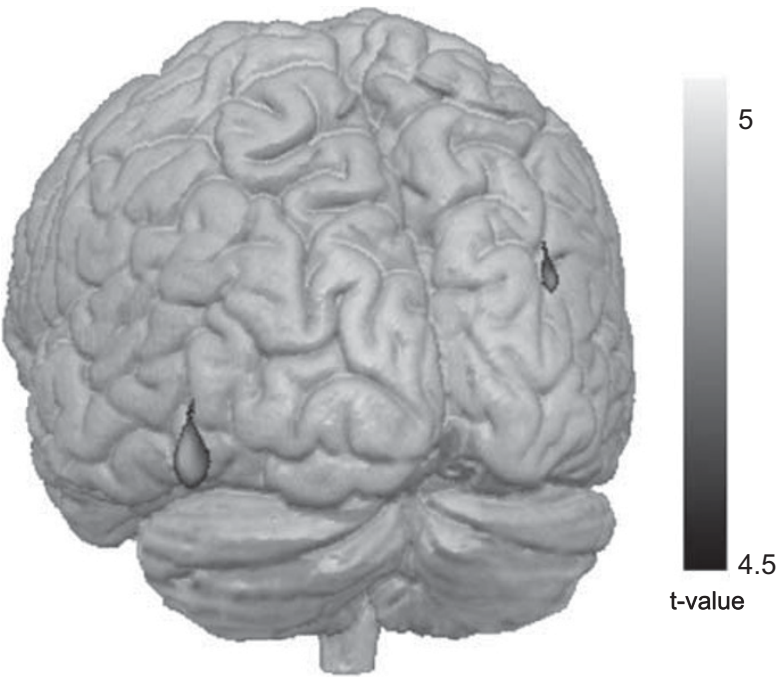

Figure I Significant decrease in gray matter volume in subjects with a history of deprivation $(n=126)$ vs those with a history of abuse $(n=127)$ in childhood in the fusiform gyrus and middle occipital gyrus. A 3D rendering is shown with color-coded differences in blue, thresholded at $p_{F W E}<0.05$ for the $\mathrm{t}$-contrast deprivation group < abuse group. A full color version of this figure is available at the Neuropsychopharmacology journal online.

$1960 / 1100 / 4.58 / 176$, and the use of GRAPPA parallel imaging with an acceleration factor of 2 .

\section{Image Data Processing}

Local differences in gray and white matter volume related to group differences were studied using a voxel-based morphometry approach (Ashburner and Friston, 2000). For this analysis, T1-images were processed with the default procedures and routines of the VBM8 toolbox (http://dbm.neuro. uni-jena.de/vbm/) within SPM8 (http://www.fil.ion.ucl.ac. $\mathrm{uk} / \mathrm{spm} /$ ). Using a unified model, T1-images were bias-field corrected, segmented into GM, white matter, and cerebrospinal fluid, and registered with the standard MNI152 brain template by way of high-dimensional DARTEL warping (Ashburner, 2007). The resulting images were modulated by the non-linear part of their DARTEL warp field and smoothed with an 10-mm FWHM Gaussian smoothing kernel, providing for an analysis of relative differences in regional gray and white matter volume, corrected for individual brain size. A detailed description of the protocol can be found in the VBM8 manual (http://dbm.neuro.unijena.de/vbm8/VBM8-Manual.pdf).

\section{Data Analysis}

For the main analyses, group was entered as a factor and age, sex, MR scanner field strength, and PANAS-negative scale were entered as covariates. These covariates were added to ensure that small differences between our matched groups would not influence our findings. For the interaction analyses, an additional product term (group $\times$ sex) was added to the fully adjusted model. When significant, the interaction effects were further explored by constructing a new model per sex. Group was then entered as a factor and age, scanner, and PANAS-negative scale served as covariates.
All statistical tests were family-wise error rate (FWE) corrected for multiple comparisons across the entire brain $\left(p_{\mathrm{FWE}}<0.05\right)$ or across all voxels in a region of interest $(\mathrm{ROI})$ using a small volume correction (SVC; $\left.p_{\text {SVC }}<0.05\right)$. SVCs were only applied in the follow-up analysis, when the interaction analysis returned clusters that were significant on a whole-brain level (initial $p_{\mathrm{FWE}}<0.05$ ). ROIs were based on a standardized neuroanatomical atlas and used for post hoc small volume correction (Tzourio-Mazoyer et al, 2002). Mean beta values of significant clusters were extracted for visualization purposes only, using the SPM toolbox MarsBar, version 0.42 (http://marsbar.sourceforge.net).

All other statistical analyses were performed using PASW Statistics 19. Significance level was $p=0.05$. Comparisons between the three groups were made using one-way ANOVAs. When comparing men with women or when directly comparing two CA groups, independent $t$-tests and chi-square tests were used for respectively continuous and categorical variables.

\section{RESULTS}

The matched groups did not differ with respect to mean age, sex, educational level, negative affect scores, total brain volume, and MRI field strength (Table 1). In addition, with the exception of total brain volume (larger in males), there were no significant sex differences for these variables. We found no indications for group differences in TR $\left(\chi^{2}=3.08\right.$, $\mathrm{df}=6, p=0.80)$ and $\mathrm{TE}\left(\chi^{2}=24.05, \mathrm{df}=18, p=0.15\right)$. Also, there were no significant group $\times$ gender interaction effects in $\mathrm{TR}(\mathrm{F}=1.855, \mathrm{df}=1, p=0.174)$ and $\mathrm{TE}(\mathrm{F}=0.015, \mathrm{df}=1$, $p=0.904)$. Finally, there were no significant group differences within the separate groups of men and women (Supplementary Table S2-AB).

\section{Specific Effects of CA}

In an initial analysis across both men and women, no differences in GM volume between either of the CA groups were found when compared with the control group. In addition, we found no significant main effect of CA (abuse group and deprivation group combined) on GM volume when compared with the control group (whole-brain $\left.p_{\text {FWE }}>0.05\right)$. However, in line with a divergent effect of different CA subtypes, we observed differences between the two CA groups in the left fusiform gyrus and the right middle occipital gyrus, whereby subjects in the deprivation group had smaller GM volumes than subjects in the abuse group (whole-brain $p_{\mathrm{FWE}}<0.05$; Figure 1, Supplementary Table S3, Supplementary Figure S1). When repeating this analysis without correction for negative affect, our two clusters were still significant $\left(p_{\mathrm{FWE}}<0.05\right)$, suggesting that negative affect did not account for these group differences.

\section{Interactions with Sex}

Next, we tested the hypothesis that sex interacts with CA effects on GM differences. We found a significant interaction between CA history and sex in the right visual posterior precuneal region (CA groups $<$ control group, $p_{\mathrm{FWE}}=0.001$ ) (Margulies et al, 2009) (S3). Post hoc analyses indicated that women with a history of CA (independent of type) had 


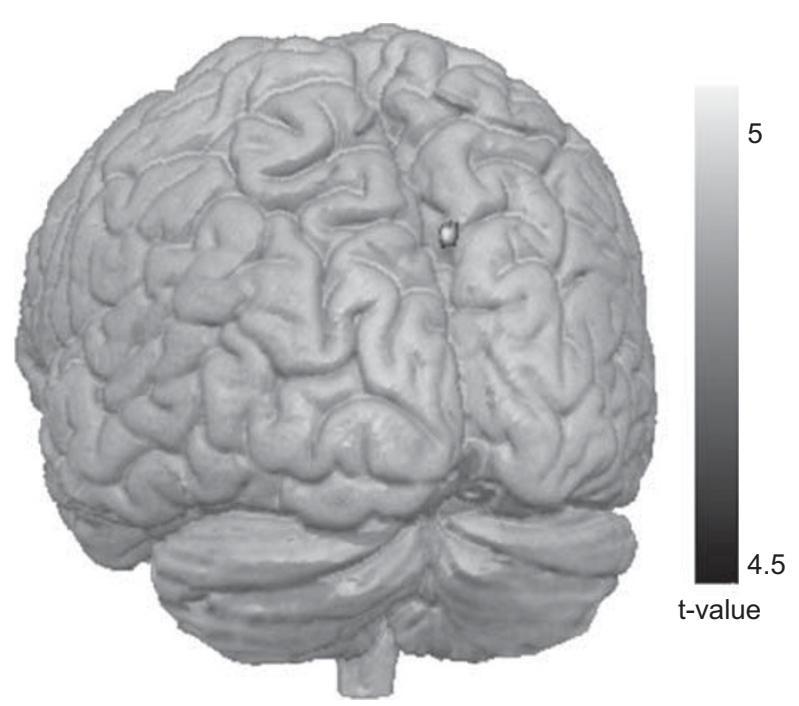

Figure 2 Significant decrease in gray matter volume in women with a history of childhood deprivation or abuse $(n=143)$ vs controls $(n=72)$ in the right inferior visual posterior precuneal region. A 3D rendering is shown with color-coded differences in blue, thresholded at $p_{\mathrm{FWE}}<0.05$ for the t-contrast CA groups < control group. A full color version of this figure is available at the Neuropsychopharmacology journal online.

smaller GM volume in this region than women in the control group $\left(p_{\mathrm{FWE}}=0.006\right)$ (Figure 2, Supplementary Table S4A, Supplementary Figure S2A), whereas in men no significant differences were found.

A second significant group $\times$ sex interaction was found in the left postcentral gyrus (S3). Here, male subjects from the deprivation group had significantly smaller GM volumes in the bilateral postcentral gyrus than men in the abuse group $\left(p_{\mathrm{SVC}}<0.05\right)$ (Figure 3 , Supplementary Table S4B, Supplementary Figure S2B).

\section{Correction for Later Abuse}

As $34 \%$ of the subjects in the abuse group also reported having experienced abuse after the age of 16 years, we tested whether our findings could be due to the events that occurred later in life. To this end, we added the binary variable of later abuse (yes or no) as a covariate to our fully adjusted model. Later abuse was defined as any of the items indicating 'abuse' in Table 1 occurring after age 16 years. After this correction, only the sex-related differences in GM volume in the postcentral gyrus (men with deprivation $v s$ men with abuse) were no longer detectable. All other findings remained significant (see for all data Supplementary Table S5).

\section{Comparison of Volumes of Interest}

Surprisingly, we did not find any effects on hippocampus and amygdala volume using our voxel-wise, brain-wide analysis, despite previous evidence of CA-related psychopathology involving these regions (eg, Hanson et al, 2015). Therefore, we decided to revisit these null findings by conducting a volumetric analysis using an automated segmentation technique (Supplementary Materials and Methods). Here, we replicated our previous finding that there were no differences in bilateral hippocampus or

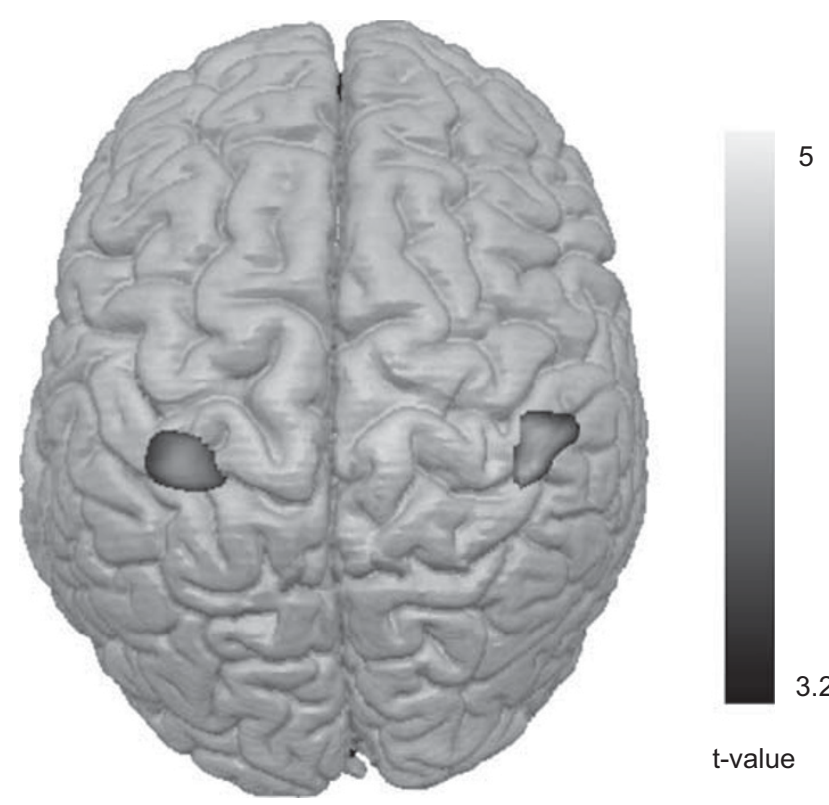

Figure 3 Significant decrease in gray matter volume in men with a history of childhood deprivation $(n=52)$ vs men with a history of abuse $(n=58)$ in the postcentral gyrus. A $3 \mathrm{D}$ rendering is shown with color-coded differences in blue, for the t-contrast deprivation group < abuse group thresholded at Puncorrected $<0.00 \mathrm{I}$ and masked with an anatomical mask for the bilateral postcentral gyrus. A full color version of this figure is available at the Neuropsychopharmacology journal online.

amygdala volume between the three groups $(p>0.1)$ (Supplementary Table S6).

\section{DISCUSSION}

The present data suggest that even in a healthy sample, subtle CA-specific alterations in GM structure can be found. In line with the hypothesis of specific effects of CA subtypes, specific associations were found in the fusiform gyrus and middle occipital gyrus, whereby subjects in the deprivation group revealed significantly smaller GM volumes than those belonging to the abuse group. In addition, sex-specific differences in somatosensory integration areas were found.

The fusiform gyrus and middle occipital gyrus are brain regions responsible for visual processing and multimodal integration (Kravitz et al, 2011). More specifically, these areas have been associated with face perception (Kanwisher et al, 1997) and scene perception (Dilks et al, 2013), respectively. Enhanced activity in the fusiform gyrus has also been related to the processing of personally familiar faces compared with faces of strangers, which suggests that its function may go beyond simple face perception (Gobbini et al, 2004). Previous studies reporting structural changes in visual processing areas in relation to CA are scarce. One study showed reduced cortical thickness in V2 and the left occipital pole after witnessing domestic violence in childhood (Tomoda et al, 2012). Another study found that subjects had smaller GM volumes in the fusiform and middle occipital gyrus after sexual abuse in childhood (Tomoda et al, 2009). Although these previous studies all report smaller GM volumes after abuse, our data revealed smaller GM volumes in subjects that had experienced deprivation in 
childhood. One explanation for this inconsistency could be that, whereas high-threat environments in humans are often well defined and investigated, the amount of deprivation in these same environments is usually unclear (Sheridan and McLaughlin, 2014), and the contribution of deprivation to the findings in abuse studies is therefore often unknown. Possibly, some of the effects that have been attributed to abuse in the past could be in fact related to deprivation. Notably, from animal models we know that deprivation in early development can have extensive consequences on neural development (Diamond et al, 1975). Studies in the field of perceptual neuroscience have shown that early visual deprivation in animals and humans leads to radical structural changes, resulting from the reduction of synapses in the primary visual cortex (Leporé et al, 2010; O'Kusky, 1985). In humans, widespread reductions in cortical thickness in regions including the fusiform gyrus and precuneus have been found in Romanian children that had experienced pronounced early-life deprivation, mediating problems with inattention and/ or impulsivity (McLaughlin et al, 2014b; Sheridan et al, 2012).

Of note, in our subjects, experiences of deprivation all occurred within the context of the subject's family, whereas abuse could also occur outside of the family context, which could suggest a possible bias toward more severe experiences in the deprivation group (Edwards et al, 2003). We tested this hypothesis by dividing the abuse group into two subgroups: one with subjects who reported abuse within the family and one with subjects who exclusively reported abuse outside their family. Importantly, only a minority (35\%) of the subjects in the abuse group exclusively experienced abuse outside their family. Moreover, additional analyses showed that the subjects within the abuse group that had either experienced abuse within or outside their family did not significantly differ from each other in GM volume (whole-brain $p_{\text {FWE }}>0.05$ ), hippocampus, or amygdala volume (Supplementary Results). This suggests that the context of the abuse did not significantly influence brain structure in our sample.

We also found two sex-specific effects in somatosensory integration areas. The visual posterior precuneal region was affected in women, both after abuse and deprivation in childhood. This region has been found to have close functional connectivity with the fusiform gyrus and also represents a transition from occipital to limbic connectivity in the precuneus (Margulies et al, 2009). It is therefore a cortical area representing the interplay of sensory input and the processing of emotions. Notably, larger functional connectivity of the precuneus with the hippocampus and ACC in women than men could be one mechanism leading to sex-specific alterations in precuneal GM structure after CA (Zhang and Li, 2012). One other study in women found reduced cortical thickness in this area after experiencing childhood emotional abuse (Heim et al, 2013). In addition, the precuneus has been found to have an important role in the mediation of grief in women (Gündel et al, 2003). Of note, thickness of the precuneal cortex has been shown to be inversely correlated with sensitivity to interpersonal rejection in a large sample of healthy college students, suggesting that this anatomical difference may underlie differences in emotion processing in a healthy population (Sun et al, 2014).
The postcentral gyrus was the second region showing sex-specific changes, where males had smaller GM volumes after a history of deprivation than male subjects with a history of abuse. However, as this effect disappeared after correction for more recent abuse, this finding may not be specific for childhood abuse. In the light of previous findings in a female clinical sample, we had expected to find smaller GM volumes in the somatosensory cortex related to a history of sexual abuse (Heim et al, 2013). Although these findings seem contradictory, they in fact highlight the importance of defining sex-specific pathways in the processing of stressful stimuli. Different pathways could be involved in the processing of fearful events in men and women and therefore lead to different neural correlates (Everaerd et al, 2012). For example, in men only, the postcentral gyrus seems to be involved in the processing of fearful faces (Weisenbach et al, 2014), which could be one potential factor of interest in the interaction we found in our sample.

From a developmental perspective, sex differences in neural correlates of CA may occur because of a number of reasons, such as the influence of gonadal hormones and different 'sensitive' time windows during neural development in boys and girls (Crozier et al, 2014; Lenroot et al, 2007; Young and Korszun, 2010). For example, with respect to our data, a recent study found that connectivity between amygdala sub-regions, the precuneus, and the postcentral gyrus shows an age by sex interaction in adolescents (Alarcón et al, 2015). This could be one potential mechanism leading to sex-specific effects in these regions dependent on the timing of adverse events. In psychopathology, these sex differences may contribute to the differences in prevalence of different psychiatric disorders, such as more depression in women and more impulse control disorders in men (Kessler et al, 1993, 2006).

Remarkably, we did not find any differences in GM structure of other areas that have often been associated with CA, such as the amygdala, hippocampus, ACC, and prefrontal cortex (Dannlowski et al, 2012; Edmiston et al, 2011; Hanson et al, 2015). One explanation for this missing finding could be that our population consisted of particularly young, highly educated, and healthy subjects with no psychiatric consequences of their early adverse life environments. Consequently, these subjects might be a particularly resilient subset of young adults exposed to childhood stress, and differ in that aspect from patient populations that are traditionally examined for consequences of CA (Lim et al, 2014), or from subjects in longitudinal studies involving severe adversity such as institutionalization (Zeanah et al, 2003). Furthermore, it has been suggested that differences in hippocampal volume after CA are not yet visible in late adolescence (Tottenham and Sheridan, 2009). In addition, we excluded subjects with threatening life events after adolescence, in contrast to most other studies. As a consequence, the differences owing to CA within our young, resilient population may be difficult to detect. However, we expect that the differences we were able to detect are particularly robust and representative of consequences of CA in a healthy population, although our results necessitate replication in different cohorts.

Potentially limiting and maybe related to the healthy nature of our subjects, we found the most pronounced differences between the two CA groups and not when 
comparing these groups with a control group. One speculative explanation could be that deprived children are significantly under-stimulated as compared with their peers, which in turn may lead to an underdeveloped visual association cortex (Sheridan and McLaughlin, 2014). In contrast, experience with high-threat situations in childhood may increase GM volumes in these areas, possibly leading to superior vigilance for fearful and sad facial expression after maltreatment (Leist and Dadds, 2009). Although this reasoning is highly speculative, our findings do stress the importance of specifically assessing the nature of adverse childhood experiences, and highlight the need for future studies looking into their mechanistic underpinnings.

Importantly, we used a large sample that consisted of healthy subjects without any confounding variables such as somatic or psychiatric disease and with a substantial subset of male participants. In addition, we used a questionnaire that allowed us to correct for possibly confounding contributions of more recent adverse events (Brugha et al, 1985). Limitations are the use of a self-reported questionnaire, the absence of information on the exact timing and frequency of the events during development, and the crosssectional design. In addition, it is possible that not all adverse childhood events were reported by all subjects, because of the sensitive nature of this information.

To conclude, we found partly sex-dependent differences in GM volumes between three well-controlled groups of healthy, young adults that had experienced environments of either abuse or deprivation in childhood and a control group. These differences could potentially give rise to specific changes in mental well-being or symptoms of psychopathology (Edmiston et al, 2011; Hanson et al, 2015). Future studies are needed to expand the current findings by examining behavioral consequences of the observed structural differences in even larger, similarly well-selected populations, such as possible differences in processing of facial expressions or other salient information, differences in parent-child attachment, somatic symptoms in psychiatric disease or somatic disease, all of which have been associated with CA in the past (Cuijpers et al, 2011; Leist and Dadds, 2009; McGoron et al, 2012). Finding specific behavioral consequences of different types of CA could provide insight into the emergence of distinct neurodevelopmental trajetories, and into potential development of specific psychiatric symptoms and disease in some vulnerable individuals.

\section{FUNDING AND DISCLOSURE}

The authors have no competing financial interests in relation to the work described. BF has received speaker fees from Merz and IT has received speaker fees from Servier and Lundbeck. The remaining authors declare no conflict of interest.

\section{ACKNOWLEDGMENTS}

We wish to thank all persons who kindly participated in the $\mathrm{BIG}$ research and the BIG team for their help with data processing. This work makes use of the BIG database, first established in Nijmegen, The Netherlands, in 2007. This resource is now part of the Cognomics Initiative (www.cognomics.nl), a joint initiative by researchers of the
Donders Centre for Cognitive Neuroimaging, the Human Genetics and Cognitive Neuroscience departments of the Radboud University Medical Center and the Max Planck Institute for Psycholinguistics in Nijmegen. The Cognomics Initiative is supported by the participating departments and centers and by external grants, ie, from the Biobanking and Biomolecular Resources Research Infrastructure (Netherlands) (BBMRI-NL), the Hersenstichting Nederland, the Netherlands Organization for Scientific Research (NWO), and from the National Institutes of Health (NIH) Consortium grant U54 EB020403, supported by a cross-NIH alliance that funds Big Data to Knowledge Centers of Excellence.

\section{REFERENCES}

Alarcón G, Cservenka A, Rudolph MD, Fair DA, Nagel BJ (2015). Developmental sex differences in resting state functional connectivity of amygdala sub-regions. NeuroImage 115: 235-244.

Ashburner J (2007). A fast diffeomorphic image registration algorithm. NeuroImage 38: 95-113.

Ashburner J, Friston KJ (2000). Voxel-based morphometry-the methods. NeuroImage 11: 805-821.

Brugha T, Bebbington P, Tennant C, Hurry J (1985). The list of threatening experiences: a subset of 12 life event categories with considerable long-term contextual threat. Psychol Med 15: 189-194.

Chan SWY, Goodwin GM, Harmer CJ (2007). Highly neurotic never-depressed students have negative biases in information processing. Psychol Med 37: 1281-1291.

Crozier JC, Wang L, Huettel SA, De Bellis MD (2014). Neural correlates of cognitive and affective processing in maltreated youth with posttraumatic stress symptoms: does gender matter? Dev Psychopathol 26: 491-513.

Cuijpers P, Smit F, Unger F, Stikkelbroek Y, Have ten M, de Graaf R (2011). The disease burden of childhood adversities in adults: a population-based study. Child Abuse Neglect 35: 937-945.

Dannlowski U, Stuhrmann A, Beutelmann V, Zwanzger P, Lenzen T, Grotegerd D et al (2012). Limbic scars: long-term consequences of childhood maltreatment revealed by functional and structural magnetic resonance imaging. Biol Psychiatry 71: 286-293.

Diamond MC, Lindner B, Johnson R, Bennett EL, Rosenzweig MR (1975). Differences in occipital cortical synapses from environmentally enriched, impoverished, and standard colony rats. J Neurosci Res 1: 109-119.

Dilks DD, Julian JB, Paunov AM, Kanwisher N (2013). The occipital place area is causally and selectively involved in scene perception. J Neurosci 33: 1331-6a.

Edmiston EE, Wang F, Mazure CM, Guiney J, Sinha R, Mayes LC et al (2011). Corticostriatal-limbic gray matter morphology in adolescents with self-reported exposure to childhood maltreatment. Arch Pediatr Adolesc Med 165: 1069-1077.

Edwards VJ, Holden GW, Felitti VJ, Anda RF (2003). Relationship between multiple forms of childhood maltreatment and adult mental health in community respondents: results from the adverse childhood experiences study. Am J Psychiatry 160: 1453-1460.

Everaerd D, Gerritsen L, Rijpkema M, Frodl T, van Oostrom I, Franke B et al (2012). Sex modulates the interactive effect of the serotonin transporter gene polymorphism and childhood adversity on hippocampal volume. Neuropsychopharmacology 37: $1848-1855$.

Gilbert R, Widom CS, Browne K, Fergusson D, Webb E, Janson S (2009). Burden and consequences of child maltreatment in highincome countries. Lancet 373: 68-81.

Gobbini MI, Leibenluft E, Santiago N, Haxby JV (2004). Social and emotional attachment in the neural representation of faces. NeuroImage 22: 1628-1635. 
Gündel H, O'Connor M-F, Littrell L, Fort C, Lane RD (2003). Functional neuroanatomy of grief: an FMRI study. Am J Psychiatry 160: 1946-1953.

Hanson JL, Nacewicz BM, Sutterer MJ, Cayo AA, Schaefer SM, Rudolph KD et al (2015). Behavioral problems after early life stress: contributions of the hippocampus and amygdala. Biol Psychiatry 77: 314-323.

Heim C, Shugart M, Craighead WE, Nemeroff CB (2010). Neurobiological and psychiatric consequences of child abuse and neglect. Dev Psychobiol 52: 671-690.

Heim CM, Mayberg HS, Mletzko T, Nemeroff CB, Pruessner JC (2013). Decreased cortical representation of genital somatosensory field after childhood sexual abuse. Am J Psychiatry 170: 616-623.

Humphreys KL, Zeanah CH (2014). Deviations from the expectable environment in early childhood and emerging psychopathology. Neuropsychopharmacology 1-17 (doi:10.1038/npp.2014.165).

Kanwisher N, McDermott J, Chun MM (1997). The fusiform face area: a module in human extrastriate cortex specialized for face perception. J Neurosci 17: 4302-4311.

Kendler KS, Gardner CO (2014). Sex differences in the pathways to major depression: a study of opposite-sex twin pairs. Am J Psychiatry 171: 426-435.

Kessler RC, Coccaro EF, Fava M, Jaeger S, Jin R, Walters E (2006). The prevalence and correlates of DSM-IV intermittent explosive disorder in the National Comorbidity Survey Replication. Arch Gen Psychiatry 63: 669-678.

Kessler RC, McGonagle KA, Swartz M, Blazer DG, Nelson CB (1993). Sex and depression in the National Comorbidity Survey. I: lifetime prevalence, chronicity and recurrence. J Affect Disord 29: 85-96.

Kravitz DJ, Saleem KS, Baker CI, Mishkin M (2011). A new neural framework for visuospatial processing. Nat Rev Neurosci 12: 217-230.

Leist T, Dadds MR (2009). Adolescents' ability to read different emotional faces relates to their history of maltreatment and type of psychopathology. Clin Child Psychol Psychiatry 14: 237-250.

Lenroot RK, Giedd JN (2010). Sex differences in the adolescent brain. Brain Cogn 72: 46-55.

Lenroot RK, Gogtay N, Greenstein DK, Wells EM, Wallace GL, Clasen LS et al (2007). Sexual dimorphism of brain developmental trajectories during childhood and adolescence. NeuroImage 36: $1065-1073$.

Leporé N, Voss P, Lepore F, Chou Y-Y, Fortin M, Gougoux F et al (2010). Brain structure changes visualized in early- and late-onset blind subjects. NeuroImage 49: 134-140.

Lim L, Radua J, Rubia K (2014). Gray matter abnormalities in childhood maltreatment: a voxel-wise meta-analysis. Am J Psychiatry 171: 854-863.

Lupien SJ, McEwen BS, Gunnar MR, Heim C (2009). Effects of stress throughout the lifespan on the brain, behaviour and cognition. Nat Rev Neurosci 10: 434-445.

Margulies DS, Vincent JL, Kelly C, Lohmann G, Uddin LQ, Biswal BB et al (2009). Precuneus shares intrinsic functional architecture in humans and monkeys. Proc Natl Acad Sci 106: 20069-20074.

McGoron L, Gleason MM, Smyke AT, Drury SS, Nelson CA, Gregas MC et al (2012). Recovering from early deprivation: attachment mediates effects of caregiving on psychopathology. J Am Acad Child Adolesc Psychiatry 51: 683-693.

McLaughlin KA, Greif Green J, Gruber MJ, Sampson NA, Zaslavsky AM, Kessler RC (2012). Childhood adversities and first onset of psychiatric disorders in a national sample of US adolescents. Arch Gen Psychiatry 69: 1151-1160.
McLaughlin KA, Sheridan MA, Lambert HK (2014a). Childhood adversity and neural development: deprivation and threat as distinct dimensions of early experience. Neurosci Biobehav Rev 47C: $578-591$.

McLaughlin KA, Sheridan MA, Winter W, Fox NA, Zeanah CH, Nelson CA (2014b). Widespread reductions in cortical thickness following severe early-life deprivation: a neurodevelopmental pathway to attention-deficit/hyperactivity disorder. Biol Psychiatry 76: 629-638.

O'Kusky JR (1985). Synapse elimination in the developing visual cortex: a morphometric analysis in normal and dark-reared cats. Brain Res 354: 81-91.

Rijpkema M, Everaerd D, van der Pol C, Franke B, Tendolkar I, Fernández G (2011). Normal sexual dimorphism in the human basal ganglia. Hum Brain Mapp 33: 1246-1252.

Sheridan MA, Fox NA, Zeanah CH, McLaughlin KA, Nelson CA (2012). Variation in neural development as a result of exposure to institutionalization early in childhood. Proc Nat Acad Sci 109: 12927-12932.

Sheridan MA, McLaughlin KA (2014). Dimensions of early experience and neural development: deprivation and threat. Trends Cogn Scie 18: 580-585.

Sun J, Li H, Li W, Wei D, Hitchman G, Zhang Q et al (2014). Regional gray matter volume is associated with rejection sensitivity: a voxel-based morphometry study. Cogn Affect Behav Neurosci 14: 1077-1085.

Tomoda A, Navalta CP, Polcari A, Sadato N, Teicher MH (2009). Childhood sexual abuse is associated with reduced gray matter volume in visual cortex of young women. Biol Psychiatry 66: 642-648.

Tomoda A, Polcari A, Anderson CM, Teicher MH (2012). Reduced visual cortex gray matter volume and thickness in young adults who witnessed domestic violence during childhood. PLoS One 7: e52528.

Tottenham N, Sheridan MA (2009). A review of adversity, the amygdala and the hippocampus: a consideration of developmental timing. Front Hum Neurosci 3: 68.

Tzourio-Mazoyer N, Landeau B, Papathanassiou D, Crivello F, Etard O, Delcroix N et al (2002). Automated anatomical labeling of activations in SPM using a macroscopic anatomical parcellation of the MNI MRI single-subject brain. NeuroImage 15: 273-289.

van Harmelen A-L, van Tol M-J, van der Wee NJA, Veltman DJ, Aleman A, Spinhoven P et al (2010). Reduced medial prefrontal cortex volume in adults reporting childhood emotional maltreatment. Biol Psychiatry 68: 832-838.

Watson D, Clark LA, Tellegen A (1988). Development and validation of brief measures of positive and negative affect: the PANAS scales. J Personality Soc Psychol 54: 1063-1070.

Weisenbach SL, Rapport LJ, Briceno EM, Haase BD, Vederman AC, Bieliauskas LA et al (2014). Reduced emotion processing efficiency in healthy males relative to females. Soc Cogn Affect Neurosci 9: 316-325.

Young E, Korszun A (2010). Sex, trauma, stress hormones and depression. Mol Psychiatry 15: 23-28.

Zeanah CH, Nelson CA, Fox NA, Smyke AT, Marshall P, Parker SW et al (2003). Designing research to study the effects of institutionalization on brain and behavioral development: the Bucharest Early Intervention Project. Dev Psychopathol 15: 885-907.

Zhang S, Li C-SR (2012). Functional connectivity mapping of the human precuneus by resting state fMRI. NeuroImage 59: $3548-3562$. 